\title{
Fractal generation method based on asymptote family of generalized Mandelbrot set and its application
}

\author{
Shuai Liu ${ }^{a}$, Zheng Pan ${ }^{a}$, Weina Fu' ${ }^{b}$ Xiaochun Cheng ${ }^{\mathrm{c}, *}$ \\ ${ }^{a}$ College of Computer Science, Inner Mongolia University, Hohhot, China. \\ ${ }^{b}$ College of Computer and Information Engineering, Inner Mongolia Agricultural University, Hohhot, China. \\ ${ }^{c}$ Department of Computer Science, Middlesex University, London, UK.
}

Communicated by X. J. Yang

\begin{abstract}
Generalized Mandelbrot set ( $k-M$ set) is the basis of fractal analysis. This paper presents a novel method to generate $k-M$ set, which generates $k-M$ set precisely by constructing its asymptote family. Correctness of the proposed method is proved as well as computational complexity is researched. Further, application of the generation method is studied, which is used to analyze distribution of boundary points and periodic points of k-M set. Finally, experiments have been implemented to evaluate the theoretical results. (C)2017 All rights reserved.
\end{abstract}

Keywords: Fractal generating method, generalized Mandelbrot set, asymptote family, boundary point, periodic point. 2010 MSC: 37F10.

\section{Introduction}

Mandelbrot proposed M set in 1982 [20], which was defined by iteration function $z \leftarrow z^{2}+c$ with initial value $z=0$. Then, $k-M$ set was defined by iteration function $z \leftarrow z^{k}+c$ also with initial value $z=0$. Thus we can see that the original $M$ set is $2-M$ set.

The iteration function $z \leftarrow z^{k}+c$ can be applied iteratively. Fractal of $k-M$ set can be researched with the set $C$ of all constant $c$ that will not make $z$ to iterate to infinite [9]. The following (1.1) is a definition of $k-M$ set when we define $f_{c}^{n}(z)=\left(f_{c}^{n-1}(z)\right)^{k}+c$ and $f_{c}^{0}(z)=0$.

$$
k-M \operatorname{set} C=\left\{c \mid \lim _{n \rightarrow \infty} f_{c}^{n}(0) \neq \infty\right\} .
$$

$k-M$ set has been researched with its fractal visualization. There are two main fractal generation methods. One is iterated function system (IFS), the other is escape time algorithm (ETA).

Affine transformation is the basic idea of IFS, which was firstly presented by Hutchinson [12]. It applies self-similarity between local and global structures of measured geometrical object and computes

\footnotetext{
*Corresponding author

Email address: $\mathrm{x}$. cheng@mdx.ac.uk (Xiaochun Cheng)
} 
transformation parameters between its local and global structures. IFS generates fractals by applying these transform parameters iteratively.

Though iterative function system (IFS) is the direct way to define fractals, escape time algorithm (ETA) has been applied to create images of complex fractals, whose parameters can not be easily computed. ETA applies an escape threshold and a maximum of iteration times to create iteration trajectories approximately. ETA iteratively generates every point in the displayed area when the computational modulus is not larger than the given escape threshold and when the iteration times are not larger than the given maximum of iteration times. ETA gives different colors to generated points in different iteration times. There are many versions of ETA. This paper discusses the following version of ETA $[9,12,20]$.

Algorithm 1.1. Escape Time Algorithm (ETA)

Let $\mathrm{N}=$ modulus threshold, $\mathrm{M}=$ iteration time threshold, $\mathrm{f}_{\mathrm{c}}(z)=z^{\mathrm{k}}+\mathrm{c}$ is the iteration function.

Step 1. For each point $\mathrm{c}$ in display area do

$\mathrm{T}_{\mathrm{c}}=0 / /$ Initialization of the number of iteration times.

$\mathrm{N}_{\mathrm{c}}=\mathrm{c} / /$ Initialization of the modulus value.

While $\mathrm{T}_{\mathrm{c}}<M$ and $\left|\mathrm{N}_{\mathrm{c}}\right| \leqslant \mathrm{N}$

$\mathrm{T}_{\mathrm{c}} \leftarrow \mathrm{T}_{\mathrm{c}}+1$ and $\mathrm{N}_{\mathrm{c}} \leftarrow \mathrm{f}_{\mathrm{c}}\left(\mathrm{N}_{\mathrm{c}}\right)$ / / Iteration until reaching end condition.

Step 2. Color point $c$ where $T_{c} \geqslant M$.

By this way, $\mathrm{k}-\mathrm{M}$ set $=\mathrm{c} \mid$ ciscolored.

ETA has been improved. Liu et al. composed continuous iteration area with the same iteration center of $k-M$ set to accelerate ETA for k-M set generation [19]. Yuan et al. presented a new ETA for Julia set generation [31]. Our team presented a new ETA analyzing relation among iteration points to reduce computation of classic ETA [14]. Then, we implemented ETA to distributed and cloud computation environments to speedup [17, 18].

Though ETA and its derivative algorithms have been widely applied in fractal research, ETA is essentially an approximate algorithm because ETA needs two given thresholds which are iteration time threshold (parameter $M$ in Algorithm 1.1) and modulus threshold (parameter $N$ in Algorithm 1.1). Different parameters generate different fractals. ETA does not give the precise escape threshold $\mathrm{N}$ or the outer structure of $M$-set.

On the other hand, characteristics of k-M set also need to be researched. Many researchers have studied this area. Huang presented a method to compute periodic orbits of $k-M$ set for positive integer $k$ by constructing polynomial equations [11]. Then, Chen and Shirriff researched basic structure of $k-M$ set for complex exponent $k[7,25]$. Meantime, Gujar and Dhurandhar explored basic structure of $k-M$ set for real exponent $k[8,10]$. Later, Wang researched divisor periodic point of $k-M$ set [30]. Pastor et al. researched periodic and chaotic regions of k-M set [24] and Negi and Rani studied midgets of superior $M$ set [23]. We presented a research result on ETA for $k-M$ set with real exponent $k$ [16]. Andreadis and Karakasidis studied numerical approximation of $k-M$ set [1].

Recently, Barral et al. studied cascade of Mandelbrot set in physics and showed its importance [4]. Buraczewski et al. studied Mandelbrot set cascades in multidimensions [5]. Ashish et al. researched features of Mandelbrot set in Noor orbit [3]. Tiozzo computed topological entropy and dimensions of sections of Mandelbrot set [28]. Andreadis and Karakasidis researched approximation of boundary structure in Mandelbrot set [2]. Wang et al. generated spatial Mandelbrot set in coupled map lattice [29]. Nazeer et al. computed fixed points in Julia and Mandelbrot sets [22]. Sun et al. researched some fractal properties in noised Mandelbrot set [26, 27].

But there are still more problems need to be solved in $k-M$ set, such as distribution of its boundary points and fixed/periodic points. Escape time algorithm (ETA) has been applied to generate fractal visualization of $k-M$ set in these references, to study and validate their research results. A novel fractal generating method is needed because of exist problems of classical ETA [14, 17, 18], for example to compute distribution of periodic and edge points can be computed by the presented algorithm.

This paper reports the following researches. 
1. A new fractal generation method of $k-M$ set.

2. Correctness and computational complexity is researched.

3. Fractal property is studied by using the generation method.

The remaining part of this paper is organized as following. Section 2 reports a novel fractal generation method of k-M sets, which is a precise algorithm. Section 3 reports proof of the proposed methods correctness and finiteness of the proposed method. Section 4 reports the application of the proposed method which includes analysis of distribution of periodic and boundary points from outside. Section 5 reports the experiment which is used to validate the theoretical analysis. Finally, Section 6 summarizes the main results of this paper.

In the following part of this paper, $\mathrm{k}$ is a rational number, $\mathrm{n}$ is a positive integer, $\mathrm{c}$ and $z$ are complex numbers. Rest of this paper will not declare them again.

\section{A novel fractal generation method}

\subsection{The generation method by reducing computational area}

We use Algorithm 2.1 to present our novel method as follows. Before the algorithm is presented, some symbols and functions are presented at first.

When using $A_{n}=\left|f_{c}^{n}(0)\right|$ to define modulus of iteration value of point $c$ with $n$ iteration times, polynomial function $H_{n}=\left\{c \mid A_{n}=2^{1 /(k-1)}\right\}$ is defined as closed polynomial curves in complex plane. Therefore, a closed surface $H_{n}^{*}=\left\{c A_{n} 2^{1 /(k-1)}\right\}$ is defined for each $H_{n}$ because its edge is $H_{n}$.

Then, Algorithm 2.1 is presented to generate $k-M$ set.

\section{Algorithm 2.1. NFGM (A Novel Fractal Generation Method)}

In this algorithm, flag marks, if there is any dropped point, $i_{c}$ with initial value 0 records iteration results for all points $c$ in computational area, $t$ with initial value 0 records iteration times, $C_{o_{t}}$ records the color with $t$ iteration times for all points, $C_{o_{1}}$ records the color of $k-M$ set and $D$ is the computational area.

Step 1. For all points $\mathrm{c}$ in the computational area D do

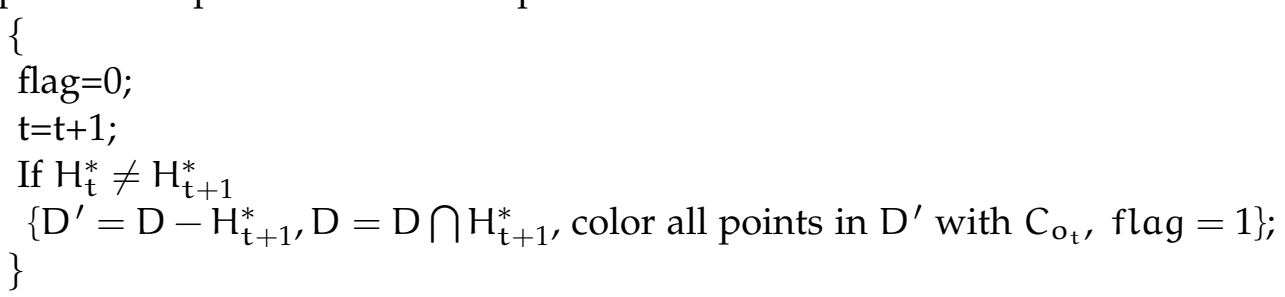

Step 2. If flag=1

goto Step 1,

else,

goto Step 1,

Color D with $\mathrm{C}_{\mathrm{o}_{1}}$ as the k-M set;

Finished.

If NFGM can generate correct k-M set, the benefit of NFGM can be presented obviously as follows.

1. First, the NFGM does not need to define thresholds of iteration time and modulus, which are defined subjectively in ETA and will cause confusion in research of $k-M$ set.

2. Secondly, convergency of NFGM seems faster than ETA with same modulus threshold. It is because that the proposed method converges faster for those special points in $k-M$ set.

3. When $k$ is a negative number, the proposed method can also generate a precise $k-M$ set. However, ETA can not generate a $k-M$ set when $k$ is negative.

\subsection{Computational time of the proposed method}

Before correctness of the proposed method is presented in Section 3, we analyze the computational time of the proposed method here. 
Theorem 2.2. Computation times of Algorithm 2.1 is no more than that of ETA with the same $\mathrm{k}-\mathrm{M}$ set.

Proof. Firstly, we prove that $M=\max t$ and $N=2^{1 /(k-1)}$ are lower bound of $M$ and $N$ in Algorithm 1.1. Case 1. Let $M<\max t$.

We know that the points in $H_{\text {max } t}^{*}$ but not in $H_{t^{\prime}}^{*}\left(t^{\prime}<t\right)$ makes $A_{M} \leqslant N$ and $A^{M+1}>N$ in generated $k-M$ set of ETA, because $\max t \geqslant M+1$. In this condition, the $k-M$ set generated by ETA has a false point $c$ which does not belong to $k-M$ set.

Case 2. Let $\mathrm{N}<2^{1 /(k-1)}$.

We know that there is at least one point $c=-2^{1 /(k-1)}$ is in real $k-M$ set but not in generated k-M set of ETA for lots of $k$ (e.g. $k=2,4$,) which means the generated $k-M$ set by ETA loses the point $c$ that belongs to $\mathrm{k}-\mathrm{M}$ set.

So we have that $M=\max t$ and $N=2^{1 /(k-1)}$ are lower bound of $M$ and $N$ in Algorithm 1.1.

Then, let $M=\max t$ and $N=2^{1 /(k-1)}$, Algorithm 2.1 and Algorithm 1.1 use the same computation times in every $\mathrm{H}_{\mathrm{n}}^{*}$ by their definitions. So the two algorithms have the same computation times.

Hence, we know ETA have computational times no less NFGM because $M=\max t$ and $N=2^{1 /(k-1)}$ are lower bound of $\mathrm{M}$ and $\mathrm{N}$ in Algorithm 1.1.

Summarizing all above mentioned, Theorem 2.2 is proved.

Further, quantitative and rigorous ratiocination for properties of NFGM is presented in following Section 3 only for positive $k$ because of Theorem 2.2.

Lemma 2.3. $\mathrm{k}-\mathrm{M}$ set contains all complex plane when $\mathrm{k}$ is negative.

Proof. Let $A_{n} \leqslant 2^{1 /(k-1)}$, we have $A_{n+1}=\left|f_{c}^{i+1}(0)\right|=\left|\left(f_{c}^{i}(0)\right)^{k}+c\right| \leqslant\left|f_{c}^{i}(0)\right|^{k}+|c|=A_{n}^{k}+A_{1}$. Thus, we know that $\lim _{i \rightarrow \infty} c_{i} \neq \infty$, because $\lim _{i \rightarrow \infty} c_{i+1} \leqslant \lim _{i \rightarrow \infty} c_{i}^{k}+c_{1}=c_{1} \neq \infty$ causes absurdity if $\lim _{i \rightarrow \infty} c_{i}=\infty$.

Lemma 2.3 means the analysis of $k-M$ set when $k$ is negative is not so meaningful. So we only discuss positive $k$ in the following sections.

\section{Properties of NFGM}

\subsection{Correctness}

The correctness of the proposed method is equal to the following Propositions 3.1, 3.2 are tautologies together.

Proposition 3.1. Iteration of every point tends to infinite, if they are not in generated $\mathrm{k}-\mathrm{M}$ set.

Proposition 3.2. Iteration of every point tends to finite, if they are in generated $\mathrm{k}-\mathrm{M}$ set.

First, we have Lemma 3.3 to prove modulus threshold of $k-M$ set.

Lemma 3.3. Modulus threshold of $k-M$ set is $2^{1 /(k-1)}$ when $k$ is positive.

Proof. To simplify the writing, use $A_{n}=\left|f_{c}^{n}(0)\right|$.

Case 1. When $A_{1}=|c|=2^{1 /(k-1)}+\varepsilon>2^{1 /(k-1)}(\varepsilon>0)$.

Let $i=1$, we have $A_{1}=2^{1 /(k-1)}+\varepsilon$.

Let $i=n$, assume $A_{n}=2^{1 /(k-1)}+n \cdot \varepsilon$.

When $i=n+1$, by using binomial theorem, we have following (3.1), which means $\lim _{n \rightarrow \infty} A_{n+1} \geqslant$ $\lim _{n \rightarrow \infty} 2^{1 /(k-1)}+(n+1) \varepsilon=\infty$.

$$
\begin{aligned}
A_{n+1}=\left|\left(f_{c}^{n}(0)\right)^{k}+c\right| \geqslant A_{n}^{k}-|c| & \geqslant\left(2^{1 /(k-1)}+n \cdot \varepsilon\right)^{k}-2^{1 /(k-1)}-\varepsilon \\
& \geqslant 2^{k /(k-1)}+k \cdot n \varepsilon \cdot\left(2^{1 /(k-1)}\right)^{k-1}-2^{1 /(k-1)}-\varepsilon \\
& \geqslant 2^{1 /(k-1)}+(n+1) \varepsilon .
\end{aligned}
$$

It means that point $c$ is not in $k-M$ set when $|c|>2^{1 /(k-1)}$. 
Case 2. When $A_{\infty} \leqslant 2^{1 /(k-1)}$.

We know $c$ belongs to $k-M$ set by its definition.

Case 3. When $A_{1}=|c|<2^{1 /(k-1)}$ and $A_{n}=2^{1 /(k-1)}+\varepsilon$. We have (3.2).

$$
\begin{aligned}
A_{n+1} & \geqslant A_{n}^{k}-A_{1}=\left(2^{1 /(k-1)}+n \cdot \varepsilon\right)^{k}-|c| \\
& \geqslant 2^{k /(k-1)}+k \cdot \varepsilon \cdot\left(2^{1 /(k-1)}\right)^{k-1}-2^{1 /(k-1)} \\
& =2^{1 /(k-1)}+2 k \cdot \varepsilon .
\end{aligned}
$$

Thus, by mathematical induction, $A_{(n+m)}=2^{1 /(k-1)}+(2 k)^{m} \cdot \varepsilon \cdot \lim _{m \rightarrow \infty} c_{n+m} \geqslant 2^{1 /(k-1)}+(2 k)^{m} \varepsilon=$ $\infty$.

As a result, $c$ is not in $k-M$ set.

However, for all points $c, c$ is not in $k-M$ set, if there exists $i$ with $\left|A_{i}\right|>2^{1 /(k-1)}$ (Case 1 and Case 3), and there exists $c$ in $k-M$ set, when $\left|A_{i}\right|=2^{1 /(k-1)}$.

So summarizing Cases 1-3, Lemma 3.3 is proved.

Lemma 3.4. $\mathrm{H}_{\mathrm{m}}^{*} \subset \mathrm{H}_{\mathrm{n}}^{*}$ when $\mathrm{n}<\mathrm{m}$.

Proof. Step 1. Proposition $c \in H_{m}^{*} \rightarrow c \in H_{n}^{*}$ is true for any $n<m$.

Let $z_{i}=f_{c}^{i}(0), c \in H_{m}^{*}$ means $A_{m}=2^{1 /(k-1)}$.

Prove by contradiction:

Assuming $A_{n}=2^{1 /(k-1)}+\varepsilon(\varepsilon>0)$ which means $c \notin H_{n}^{*}$, we have (3.3).

$$
A_{m}=\left|f_{c}^{m-n}\left(f_{c}^{n}(0)\right)\right|=\left|f_{c}^{m-n}\left(z_{\mathfrak{n}}\right)\right|=2^{1 /(k-1)} .
$$

But by using $A_{n}=2^{1 /(k-1)}+\varepsilon$, we also have (3.4).

$$
\left|f_{c}\left(z_{n}\right)\right| \geqslant A_{n}^{k}-|c|>2 \cdot 2^{1 /(k-1)}+2 k \varepsilon-2^{1 /(k-1)} \geqslant 2^{1 /(k-1)}+2 k \varepsilon .
$$

Hence, we have $\left.\mid f_{c}^{(} m-n\right)\left(z_{n}\right) \mid>2^{1 /(k-1)}$ which contradicts (3.3). It means the assumption is wrong. In brief, Step 1 is proved.

Step 2. There exists point $\mathrm{c}$ makes $\mathrm{c} \in \mathrm{H}_{\mathrm{n}}^{*}$ and $\mathrm{c} \notin \mathrm{H}_{\mathrm{m}}^{*}$.

Select any point $c$ on $H_{n}\left(\left|z_{n}\right|=2^{1 /(k-1}\right)$, we have the corresponding $z_{m}=f_{c}^{m-n}\left(f_{c}^{n}(0)\right)$.

From Step 1, we have that point $c$ in $\mathrm{H}_{\mathrm{m}}^{*}$ must satisfy (3.3). Moreover, from Step 1, we have that $\left|f_{c}\left(z_{n}\right)\right| \geqslant\left|z_{n}\right|^{k}-|c|=2 \cdot 2^{1 /(k-1)}-|c| \geqslant 2^{1 /(k-1)}$, because $|c| \leqslant 2^{1 /(k-1)}$.

When sign $>$ is accepted, we have proved Step 2, because $c \notin \mathrm{H}_{\mathrm{m}}^{*}$.

Summarizing Step 1 and 2, Lemma 3.4 is proved.

After Lemma 3.4, we follow Theorems 3.5 to prove that $H_{n}$ approaches to $k-M$ set.

Theorem 3.5. $\mathrm{H}_{\infty}^{*}$ is k-M set.

Proof. Firstly, k-M set contains all points $c$ when $c$ is in $H_{\infty}^{*}$. Otherwise, $\left|z_{\infty}\right|=2^{1 /(k-1)}$ and $c k-M$ set, and this is absurd by (1.1).

Secondly, $H_{\infty}^{*}$ contains all points $c$ when $c$ is in $k-M$ set. Otherwise, $\left|z_{\infty}\right|=2^{1 /(k-1)}$ and $c \notin H_{\infty}^{*}$ and this is absurd by Lemma 3.3.

So Theorem 3.5 is proved.

Lemma 3.4 and Theorem 3.5 prove that Proposition 3.2 is a tautology. Thus, both Propositions 3.1 and 3.2 are tautologies together, which prove correctness of the proposed method.

\subsection{Finiteness}

In order to prove the finiteness of NFGM, we have Inference 3.6 first.

Inference 3.6. There are countable intersections for $\mathrm{H}_{\mathrm{m}}$ and $\mathrm{H}_{\mathrm{n}}$ when $\mathrm{m} \neq \mathrm{n}$.

Proof. First, we follow (3.5) to compute intersections of $H_{n}$ and $H_{m}$. This is because $H_{i}=\left\{c\left|f_{c}^{i}(0)\right|=\right.$ $\left.2^{1 /(k-1)}\right\}$. 


$$
\mathrm{H}_{\mathrm{n}} \cap \mathrm{H}_{\mathrm{m}}=\left\{\mathrm{c}|| \mathrm{f}_{\mathrm{c}}^{\mathrm{n}}(0)|=| \mathrm{f}_{\mathrm{c}}^{\mathrm{m}}(0) \mid=2^{1 /(k-1)}\right\} .
$$

Thus, to simplify the proof process, we assume $m>n$ and have following (3.6).

$$
\left|z_{\mathrm{m}}\right|=\left|\mathrm{f}_{\mathrm{c}}^{\mathrm{m}-\mathrm{n}}\left(z_{\mathrm{n}}\right)=\right| z_{\mathfrak{n}} \mid=2^{1 /(k-1)} .
$$

Then, we reach following (3.7) for each $\mathrm{m}^{\prime}>\mathrm{m}$ and a large iteration time $\mathrm{p}$.

$$
\left|z_{\mathfrak{m}^{\prime}}\right|=\left|f_{\mathcal{c}}^{\mathfrak{m}^{\prime}+n-p(m-n)}\left(z_{\mathfrak{n}}\right)\right| \geqslant\left|z_{\mathfrak{n}}\right|^{k}-|c| \geqslant 2 \cdot 2^{1 /(k-1)}-2^{1 /(k-1)}=2^{1 /(k-1)} .
$$

Then, to reach the equality consistently, we have that $\left|z_{n+1}\right|=\left|z_{n}\right|=|c|=2^{1 /(k-1)}$ from Theorem 2.2.

Similarly, we have following (3.8) from proof of Lemma 3.4.

$$
\left|c^{k}+c\right|=\left|c^{k}\right|-|c| .
$$

It means $c^{k}$ has contrary vector direction with $c$. Let $c=2^{1 /(k-1)} \cdot e^{i \theta}(\theta \in[0,2 \pi])$, we have (3.9) from (3.8).

$$
\theta(k+1)=(2 h+1) \pi
$$

Solution of (3.9) is $h=i$ and $\theta=\pi /(k-1) \cdot(2 i+1)$ where $i=0, \cdots, k-1$. So there are $k$ solutions of (3.7) in the original complex plane. So solutions of (3.6) are countable because $m, n$ and $k$ are all countable.

So Inference 3.6 is proved.

Lemma 3.4 proves that $H_{n}$ are asymptote family. Theorem 3.5 proves that $H_{n}$ are asymptotic curves of k-M set, and Inference 3.6 means that these curves have only countable intersections. So we can generate $k-M$ set by these curves.

We finish Algorithm 2.1 when D is not changed. In other words, Algorithm 2.1 is finished when we reach minimal D. In this case, $\mathrm{H}_{n}-1=\mathrm{H}_{\mathrm{n}}$ is displayed area. So we prove the finiteness of Algorithm 2.1 by Inference 3.6.

Then, we present application of NFGM in fractal research as follows.

\section{Application of NFGM}

This section reports application of NFGM for k-M set, which is mainly about computation of special points by NFGM. From definition of them, eventually periodic point on edge can be solved by intersections between different $\mathrm{H}_{m}$ and $\mathrm{H}_{n}$. We have Theorem 4.1 to prove that all intersections between different $H_{m}$ and $H_{n}$ are eventually fixed or periodic point.

Theorem 4.1. Intersections between $\mathrm{H}_{m}$ and $\mathrm{H}_{n}$ are eventually periodic points on edge of $\mathrm{k}-\mathrm{M}$ set.

Proof. An intersection $c$ between $\mathrm{H}_{m}$ and $\mathrm{H}_{n}$ must suit for (4.1) when assuming $m>n$.

$$
\left|z_{m}\right|=\left|f_{c}^{m-n}\left(z_{n}\right)\right|=\left|z_{n}\right|=2^{1 /(k-1)} .
$$

Thus, for any iteration time $m^{\prime}>m$, we have following (4.2) for any $p$, which means $\lim _{m \rightarrow \infty} Z_{m} \neq \infty$.

$$
\left|z_{m^{\prime}}\right|=\left|f_{c}^{m^{\prime}+n-p(m-n)}\left(z_{n}\right)\right| .
$$

Therefore, $\mathrm{c}$ belongs to $\mathrm{k}-\mathrm{M}$ set and is an eventually periodic point.

Theorem 4.1 is proved.

Further, let $S_{n, k}$ as the number $n^{\text {th }}$ periodic point in $k-M$ set, $n 1=1<n 2<\cdots<n p$ are all divisors of $\mathrm{n}$, we have Theorem 4.2.

Theorem 4.2. $\sum_{i=1}^{p} S_{n_{i}, k}=k^{n-1}$ for postive integer $k$. 
Proof. With its definition, a periodic point can be solved by (4.3) and rewritten to (4.4).

$$
\begin{gathered}
f_{c}(0)=f_{c}^{n}(0) \quad(n>2), \\
f_{c}^{n-1}(0)=0 .
\end{gathered}
$$

Equation (4.4) is a polynomial equation with highest exponent $k^{n-2}$ and its roots are corresponding to the number of $n-1$ periodic points. So number $n^{\text {th }}$ periodic points is $k^{n-1}$. Then, we have to abandon those periodic points which belong to several periods. Assuming $n 1=1<n 2<\ldots<n p=n$ are all divisors of $n$, because for any $n_{i}$ period point $c, f_{c}^{n}(0)=f_{c}^{n-h n_{i}}(0)$ for any natural number $h$, we know that periodic points with $n_{i}$ period are all $n^{\text {th }}$ periodic points. So Theorem 4.2 is proved.

Theorem 4.2 presents intersections of $\mathrm{H}_{m}$ and $\mathrm{H}_{n}$. Specially, when $n$ is prime, we have Inference 4.3. Inference 4.3. $\mathrm{S}_{\mathrm{n}, \mathrm{k}}=\mathrm{k}^{\mathrm{n}-1}-1$ when $\mathrm{n}$ is prime.

Theorem 4.1 and Inference 4.3 explain the number of periodic points in $k-M$ set. Thus, we have following Inference 4.4 to reach all fixed points and 2-periodic points of $k-M$ set.

Inference 4.4. Zero is only fixed point of $k-M$ set, $c=e^{i \cdot[(2 a+1) \pi /(k-1)]}(a=0,1, \cdots, k-2)$ are all 2-periodic.

Proof. Let $\mathrm{n}=2,(4.4)$ is $\mathrm{c}=0$. So the root is $\mathrm{c}=0$.

Let $n=3,(4.4)$ is $c^{k}+c=0$, the roots are $c=0$ or $c=e^{i \cdot[(2 a+1) \pi /(k-1)]}$. Inference 4.4 is proved because $c=0$ is the fixed point.

Besides fixed and periodic points, the boundary points are also important in fractal analysis. When we extend (4.4) to (4.5), we have all boundary points.

$$
f_{c}^{m}(0)=f_{c}^{n}(0) \quad(m, n \geqslant 2, m \neq n) .
$$

We prove it in Theorem 4.5 as follows.

Theorem 4.5. $c$ is boundary points $\Leftrightarrow$ there exist $m, n \geqslant 2, m \neq n, f_{c}^{m}(0)=f_{c}^{n}(0)$.

Proof. (1) Assume $\mathrm{f}_{\mathfrak{c}}^{\mathrm{m}}(0)=\mathrm{f}_{\mathfrak{c}}^{\mathrm{n}}(0)$ and $m \neq n$.

We have $f_{c}^{p}(0)=f_{c}^{p-m}\left(f_{c}^{m}(0)\right)=f_{c}^{p-m}\left(f_{c}^{n}(0)\right)=f_{c}^{p-(m-n)}(0)$ which can be rewritten to $f_{c}^{p}(0)=f_{c}^{b}(0)$ by using $p=a(m-n)+b(b<m-n, p>m>n \geqslant 2)$. Thus, for any $p, f_{c}^{p}(0)$ is not infinite or iterated to zero. It means $c$ is a boundary point.

(2) Assume $c$ is a boundary point.

In [20], we know points in 1-periodic orbit of $k-M$ set are attracted by point zero and n-periodic orbits are mapped from 1-periodic orbit. So each n-periodic point attracts its $n$-periodic orbit. It means when $\mathrm{c}$ is a boundary points, we have $A_{\infty}=2^{1 /(k-1)}$ and $A_{\infty} \neq 0$. It means $c$ is an eventually periodic point of $k-M$ set [16]. In other words, there exist $m$ and $n$ make $f_{c}^{m}(0)=f_{c}^{n}(0)$.

Summarizing (1) and (2), Theorem 4.5 is proved.

Theorems 4.2-4.5 present distribution of special points in k-M set. Further, we use experiment in Section 5 to validate these conclusions.

\section{Experimental results}

\subsection{Verification of effectiveness of NFGM}

After discussion and application of NFGM, we generate examples to validate our conclusion in Sections 2-4. First, Figures 1-4 validate that modulus threshold is $2^{1 /(k-1)}$.

In fact, we know that $k-M$ set of ETA with $N=2^{1 /(k-1)}$ and $M=t$ is the same as $H_{t}^{*}$. We create Figures 1, 2 to validate our conclusions. Figures $1(\mathrm{a})-1(\mathrm{~d})$ are figures with $k=4$ and Figures $2(\mathrm{a})-2(\mathrm{~d})$ are figures with $k=8$. In [24], its authors presented a numerical approximating method of the generalized Mandelbrot sets, which is meaningful in real world like specified in reference [23]. In this paper, the asymptote curves of $k-M$ set are a helpful supplement to compute fractal properties of k-M set. Also, it can be applied in fractal description of other convergent systems. 


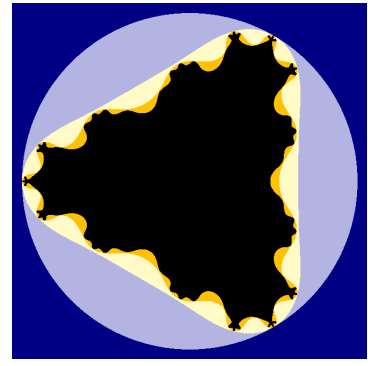

(a) $\max t=4$

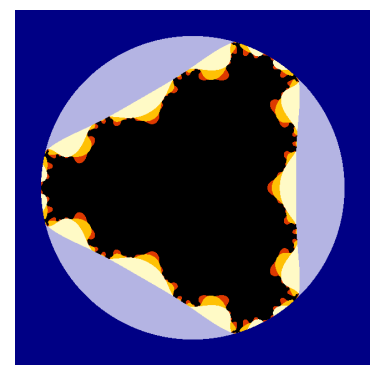

(c) $\mathrm{N}=1.1402<2^{1 / 3}$

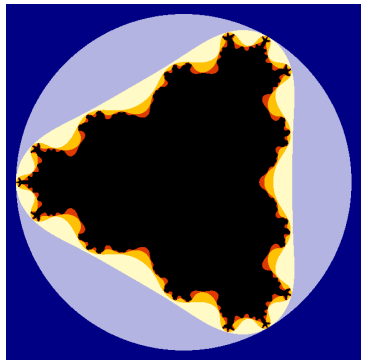

(b) $\max t=5$

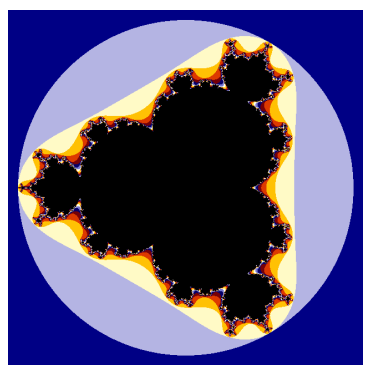

(d) 4-M set by FGMAC

Figure 1: Compare different $t$ and $N$ in $4-M$ set at display area $(-4 / 3,4 / 3) *(-4 / 3,4 / 3)$.

In Figures 1, 2, Figure 1(a) and Figure 2(a) are k-M sets by ETA with $M=4$. Figure $1(\mathrm{~b})$ and 2(b) are $k-M$ sets by ETA with $M=5$. Figure 1 (c) and Figure 2(c) are $k-M$ sets by ETA with $N<2^{1 /(k-1)}$. Fig.1(d) and Fig.2(d) are k-M sets by FGMAC (Algorithm 2.1). Area with black color in each figure is generated $k-M$ set, other colors are corresponding to different iteration times. In Figures 1-2, figure $b$ has more details than figure $a$, because it has more iteration times, $k-M$ set in figure $c$ is larger because its threshold $\mathrm{N}$ is smaller which brings wrong result, figure $d$ has most details and most accurate $k-M$ set, which means it is best in both 4 algorithms.

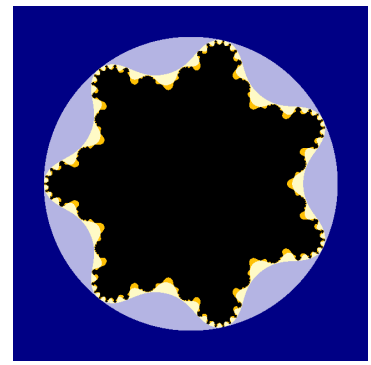

(a) $\max t=4$

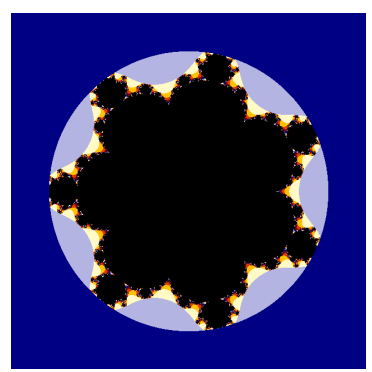

(c) $\mathrm{N}=1.0488<2^{1 / 7}$

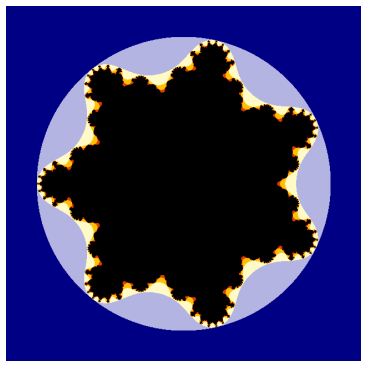

(b) $\max t=5$

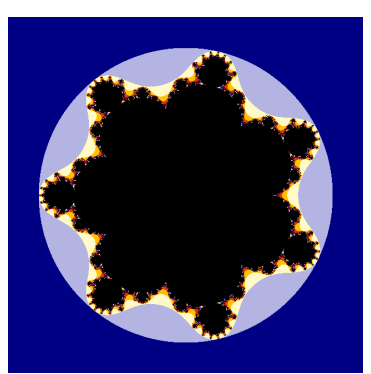

(d) 8-M set by FGMAC

Figure 2: Compare different $t$ and $N$ in $8-M$ set at display area $(-4 / 3,4 / 3) *(-4 / 3,4 / 3)$. 
In Figures 3, 4, we can see the differences between sub-figures a-d clearly. Then we can validate our theory by these figures. In Figures 3, 4, figure $b$ has orange color while figure a does not have. The orange color means iteration values of points in this area are more than $N$ by 5 times. Part of $k-M$ set in figure $a$ colors orange is figure $b$ which means figure $a$ is less accurate than figure $b$. Iteration time of figure $c$ can be seen more than figures $a-b$ from its color, but it also misses some details of $k-M$ set in edge. This is because threshold $N$ is too small that it misses those points in $k-M$ set which have large iteration values.

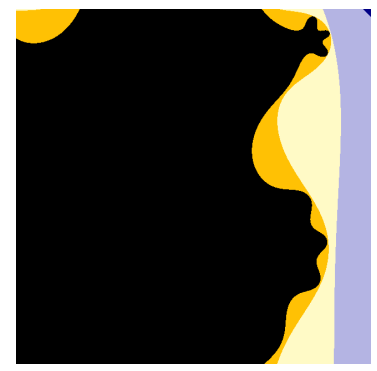

(a) $\max t=4$

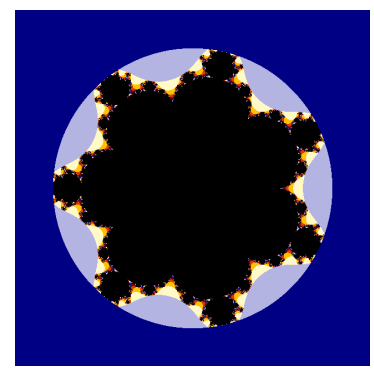

(c) $\mathrm{N}=1.1402<2^{1 / 3}$

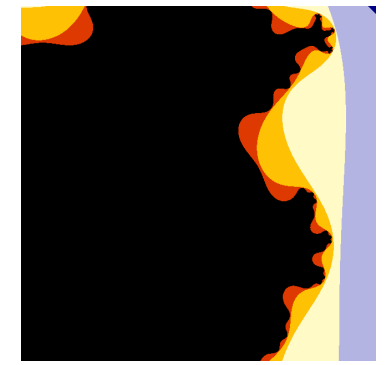

(b) $\max t=5$

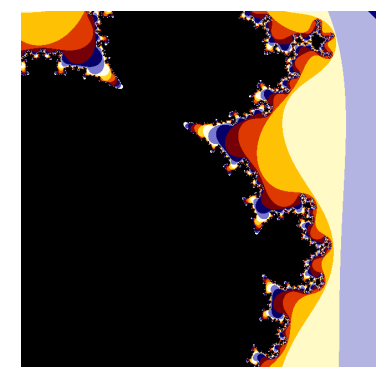

(d) 4-M set by FGMAC

Figure 3: Compare different $t$ and $N$ in $4-M$ set at display area $(0.1,0.9) *(0.1,0.9)$.

For example, orange part of Figure 2(b) contains those points which satisfy following conditions $A_{4} \leqslant N=2^{1 / 7}$ and $A_{5}>N=2^{1 / 7}$. To experiment, we get a point $c=0.44875+0.56375 i$ in $k-M$ set of Figure 2(a) which is in orange part of Figure 2(b) and find $z_{2}=0.4936+0.6209 i, z_{3}=0.5450+0.6874 i$, $z_{4}=0.6612+0.8429 i, z_{5}=1.4407+1.9863 i, z_{6}=398.69+1253.1 i, \ldots$. It is admittedly that it iterates to infinite because $\left|z_{6}\right|$ is so large, which proves k-M set in Figure 2(a) is not accurate. Also, in Figure 1(a) we get a point $c=0.2406+0.8406 i$ in $k-M$ set of Figure 1(a) which is in from orange part of Figure $1(b)$ and find $z_{2}=0.4978+0.3158 i, z_{3}=0.1637+0.9337 i, z_{4}=0.8613+0.3240 i, z_{5}=0.3347+1.5515 i$, $z_{6}=4.4292-3.9267 i$. Also, point $c$ iterates to infinite which proves $k-M$ set in Figure 1(a) is not accurate. These experiments validate Theorem 2.2 and Lemma 3.3.

\subsection{Verification of asymptote family}

Moreover, k-M sets of Figure 1(c) and Figure 2(c) are smaller than others, because the part with black color in them is smaller than others. But we choose an escape point $c=-2^{1 / 3}$ in Figure $1(\mathrm{c})$ and compute $z_{2}=z_{3}=\cdots=-c$ which means $c$ is in $k-M$ set. It means $k-M$ set in Figure 1(c) is not accurate and validates Lemma 3.3. Then, from Figure 1(d) and Figure 2(d), length of line $\theta=0$ (positive real axis) in $k-M$ set is least. All parts with different iteration times are from outside to inner, which validates Lemma 3.4. Then, k-M set in Figure 1(d) and Figure 2(d) are true k-M sets which validates Theorem 3.5. Meantime, when compare iteration times with all points in Figure 1 and Figure 2 except Figure 1(d) and Figure 2(d), it validates Theorem 2.2. Furthermore, in real application, the number of processing points reduces fast when $t$ increases. This also can be seen in Figures 1-2 (see areas with different colors).

In order to see details of these figures clearly, we enlarge their corresponding parts in Figure 3 and Figure 4. Then, Figures 5-6 present $\mathrm{H}_{1}-\mathrm{H}_{3}$ with $\mathrm{k}=4$ and 8. In these two figures, $\mathrm{H}_{1}$ colors red, $\mathrm{H}_{2}$ colors 
blue and $\mathrm{H}_{3}$ colors green. We can see edges with different colors contain each other in Figures 5-6, which validate Lemma 3.4. Then, analysis of intersections between two next edges validates Inference 3.6.

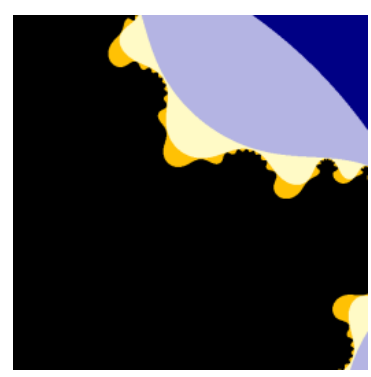

(a) $\max t=4$

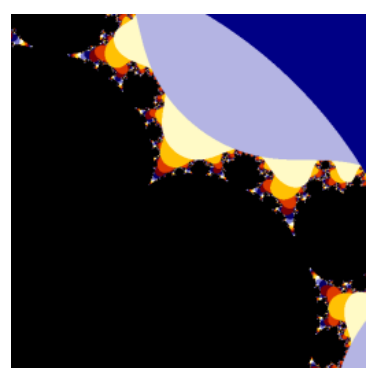

(c) $\mathrm{N}=1.0488<2^{1 / 7}$

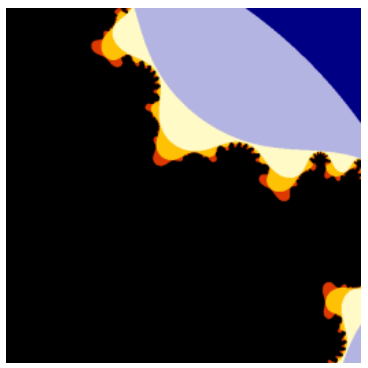

(b) $\max t=5$

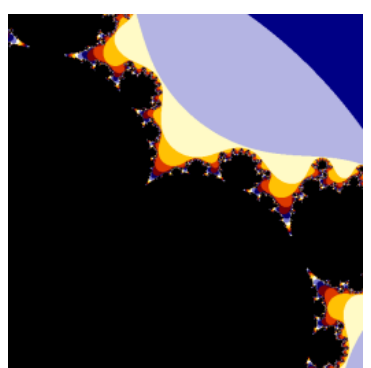

(d) 8-M set by FGMAC

Figure 4: Compare different $t$ and $N$ in $8-M$ set at display area $(0.1,0.9) *(0.1,0.9)$.

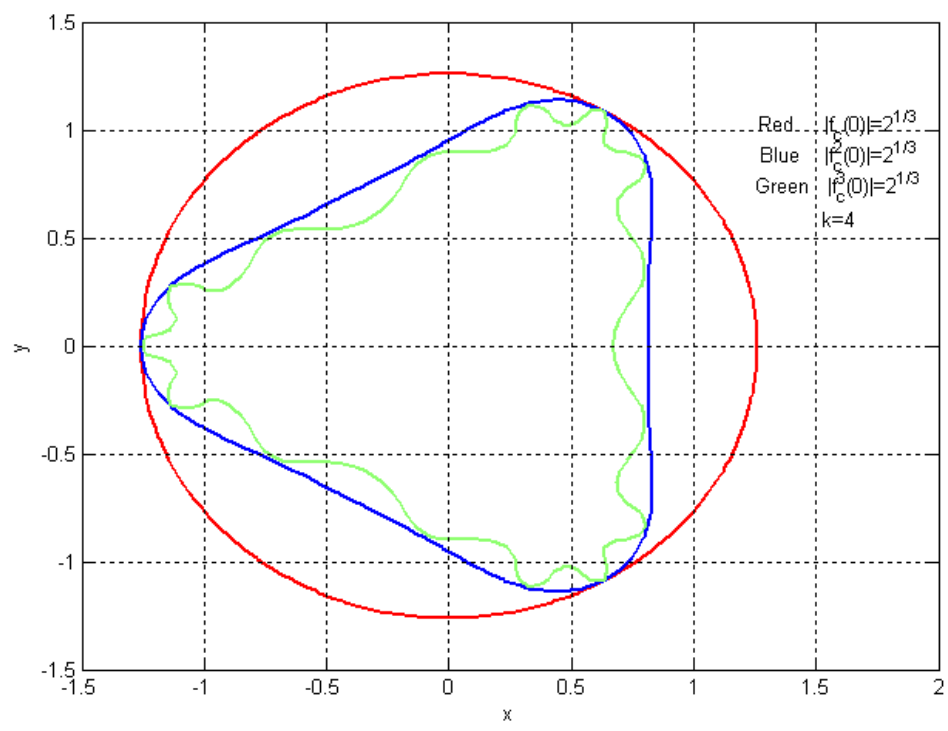

Figure 5: $H_{1}, H_{2}$ and $H_{3}$ for $k=4$.

\subsection{Verification of distribution of special points}

Finally, we have Tables 1, 2 and Figures 7-8 to validate Theorem 4.5 and Inferences 4.3-4.4, where Tables 1, 2 present numbers of boundary and periodic points for $k-M$ set and Figures 7, 8 present images of some nearby points in Tables 1,2. Centers of every sub-figures are presented in Figures 7, 8 and display area is $(0.4 * 0.4)$. 


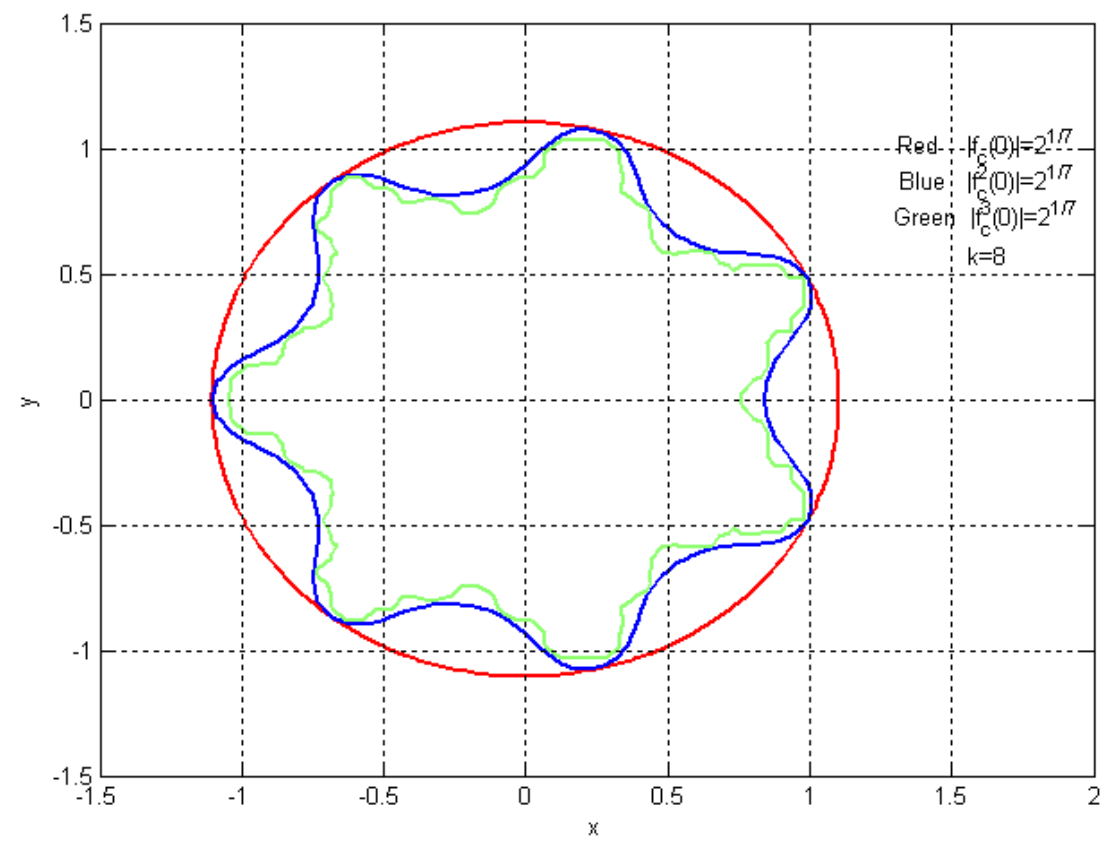

Figure 6: $H_{1}, H_{2}$ and $H_{3}$ for $k=8$.

Table 1: Some number of boundary points of k-M Set

\begin{tabular}{cc}
\hline Numbers & $\mathrm{n}=2, \mathrm{~m}=3$ \\
\hline $\mathrm{k}=2$ & 1 \\
Boundary Points & -2 \\
$\mathrm{k}=4$ & 9 \\
Boundary Points & $-1.2599,0.6300 \pm 1.0911 i,-1.0842 \pm 0.2905 i$, \\
& $0.2905 \pm 1.0842 i, 0.7937 \pm 0.7937 i$ \\
\hline
\end{tabular}

Table 2: Some number of $n$-Periodic points of $k-M$ set

\begin{tabular}{|c|c|c|c|c|}
\hline$S_{n, k}$ & $\mathrm{n}=1$ & $\mathrm{n}=2$ & $\mathrm{n}=3$ & $\mathrm{n}=4$ \\
\hline $\mathrm{k}=2$ & $\mathrm{~S}_{1,2}=1$ & $2-1=1$ & $2^{2}-1=3$ & $2^{2}-S_{1,2}-S_{2,2}=6$ \\
\hline $\begin{array}{c}\text { Computational Periodic } \\
\text { Points }\end{array}$ & 0 & -1 & $\begin{array}{c}-1.7549 \\
-0.1226 \pm 0.7449 i\end{array}$ & $\begin{array}{c}-1.3107,-1.9408 \\
0.2823 \pm 0.5301 i \\
-0.1567 \pm 1.0320 i\end{array}$ \\
\hline $\mathrm{k}=4$ & $S_{1,4}=1$ & $4-1=3$ & $4^{2}-1=15$ & $4^{3}-S_{1,4}-S_{2,4}=60$ \\
\hline Periodic Points & 0 & $1,0.5 \pm 0.8660 i$ & \multirow{3}{*}{\multicolumn{2}{|c|}{$\begin{array}{l}\text { Number of periodic points is the } \\
\text { computed number, accurate values are } \\
\text { ellipsis because the number is large } \\
6^{2}-1=35 \quad 6^{3}-S_{1,4}-S_{2,6}=210 \\
\text { Number of periodic points is the } \\
\text { computed number, accurate values are } \\
\text { ellipsis because the number is large }\end{array}$}} \\
\hline$k=6$ & $S_{1,6}=1$ & $\begin{array}{c}6-1=5 \\
-1\end{array}$ & & \\
\hline Periodic Points & 0 & $\begin{array}{c}0.8090 \pm 0.5878 i \\
-0.3090 \pm 0.9511 i\end{array}$ & & \\
\hline
\end{tabular}

In Table 1, some boundary points are computed by Theorem 4.5. Some fractal graphics are generated in Figure 8, which centers are values computed in Table 1. We find these points are really boundary points from Figure 8, which validates Theorems 4.5. Also, we find all values $c=e^{i \cdot[2 a \pi /(k-1)]}$ are boundary 
points, which validates Inference 4.4. Then, in Table 2, numbers of periodic points are computed by Theorem 4.2. Then, all periodic points are found by solving (3.8). We find number of roots of (3.8) equals to number of periodic points computed by Theorem 4.2. This validates Inference 4.3. Also, we find $c=e^{i \cdot[(2 a+1) \pi /(k-1)]}$ are all periodic points in $k-M$ set, which validates Inference 4.4 . Then, some fractal graphics are generated in Figure 7, which centers are values computed in Table 2. We find these points are really periodic points from Figure 7, which validates Theorem 4.2.

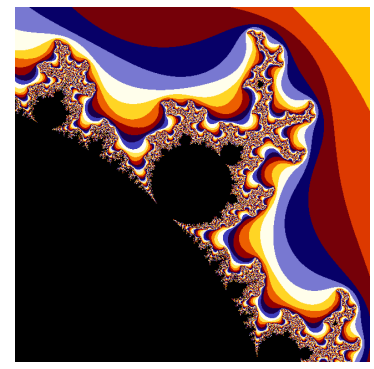

(a) $0.2823+0.5301 \mathrm{i}$

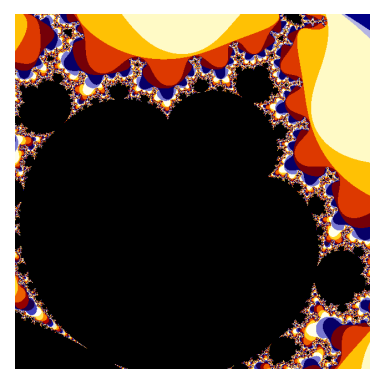

(c) $0.5+0.8660 \mathrm{i}$

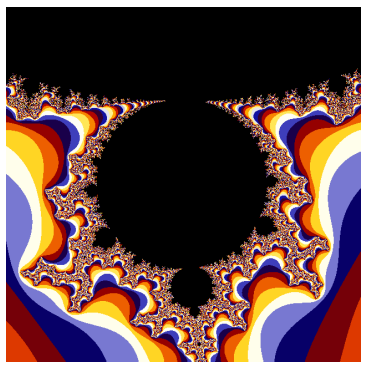

(b) $-0.1226-0.7449 \mathrm{i}$

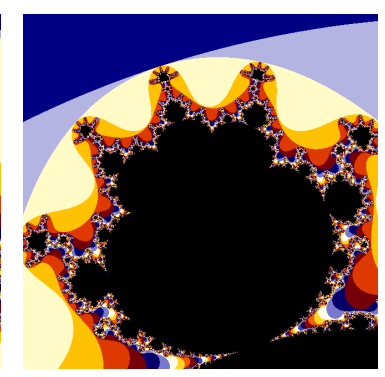

(d) $-0.3090+0.9511 \mathrm{i}$

Figure 7: Fractals and some nearby points in Table 1.

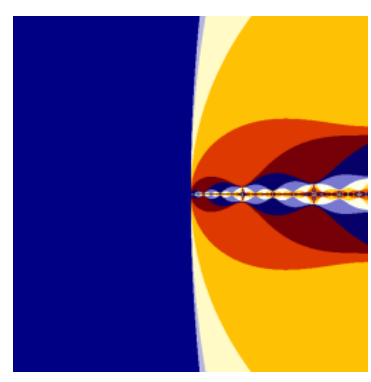

(a) -2

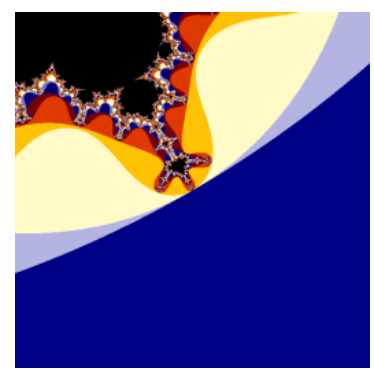

(c) $0.6300-1.0911 \mathrm{i}$

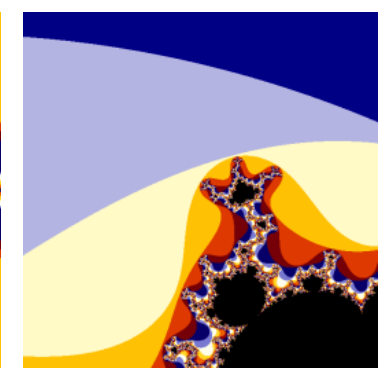

(b) $0.2905+1.0842 \mathrm{i}$

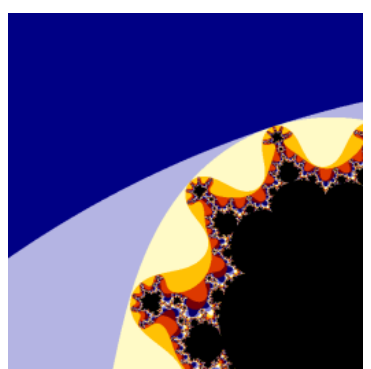

(d) $-0.4540+1.0196 \mathrm{i}$

Figure 8: Fractals and some nearby points in Table 2. 


\section{Conclusion}

This paper presented a novel fractal generating method and discussed properties of the proposed method. The proposed method was a precise algorithm with proof of its correctness and finiteness. However, the proposed method can be used to analyze the distribution of eventually periodic point, periodic and boundary points. Finally, experiment results were provided to validate the theoretical analysis.

Our future work includes following two steps. At first, we will extend results of this paper to k-M set when $k$ is a real or complex number based on references [1,2]. We will extend our theory by using modern algebra to compute the roots with maximum modulus of (4.4). Secondly, we will improve our fractal generating and visualizing method and find a suitable application based on reference [15].

Since fractal theory, feature and descriptor are widely applied in image processing, content analysis and other research domains $[6,13,21]$, our research of generalized Mandelbrot set in this paper, which is the base of fractal theory, is meaningful for fractal applications.

\section{Acknowledgment}

This research is supported by following grants: National Natural Science Foundation of China (61502254). The authors are also funded by EUFP7 WIDTH project and VALCRI project.

\section{References}

[1] I. Andreadis, T. E. Karakasidis, On numerical approximations of the area of the generalized Mandelbrot sets, Appl. Math. Comput., 219 (2013), 10974-10982. 1, 6

[2] I. Andreadis, T. E. Karakasidis, On a numerical approximation of the boundary structure and of the area of the Mandelbrot set, Nonlinear Dynam., 80 (2015), 929-935. 1, 6

[3] Ashish, M. Rani, R. Chugh, Julia sets and Mandelbrot sets in Noor orbit, Appl. Math. Comput., 228 (2014), $615-631$. 1

[4] J. Barral, A. Kupiainen, M. Nikula, E. Saksman, C. Webb, Critical Mandelbrot cascades, Comm. Math. Phys., 325 (2014), 685-711. 1

[5] D. Buraczewski, E. Damek, Y. Guivarc'h, S. Mentemeier, On multidimensional Mandelbrot cascades, J. Difference Equ. Appl., 20 (2014), 1523-1567. 1

[6] D. Casanova, J. B. Florindo, M. Falvo, O. M. Bruno, Texture analysis using fractal descriptors estimated by the mutual interference of color channels, Inform. Sci., 346 (2016), 58-72. 6

[7] N. Chen, W.-Y. Zhu, Bud-Sequence conjecture on $M$ fractal image and $M-J$ conjecture between $C$ and $Z$ planes from $z \leftarrow z^{w}+c(w=\alpha+i \beta)$, Comput. Graph., 22 (1998), 537-546. 1

[8] S. V. Dhurandhar, V. C. Bhavsar, U. G. Gujar, Analysis of z-plane fractal images from $z \leftarrow z^{\alpha}+c$ for $\alpha<0$, Comput. Graph., 17 (1993), 89-94. 1

[9] K. Falconer, Fractal geometry, Mathematical foundations and applications, Second edition, John Wiley \& Sons, Inc., Hoboken, NJ, (2003). 1, 1

[10] U. G. Gujar, V. C. Bhavsar, N. Vangala, Fractal images from $z \leftarrow z^{\alpha}+c$ in the complex z-plane, Comput. Graph., 16 (1992), 45-49. 1

[11] Y.-N. Huang, Global analysis of the Mandelbrot set and the general Mandelbrot set, China Sci. A, 8 (1991), 823-830. 1

[12] J. E. Hutchinson, Fractals and self-similarity, Indiana Univ. Math. J., 30 (1981), 713-747. 1

[13] W.-L. Lee, Y.-C. Chen, Y.-C. Chen, K.-S. Hsieh, Unsupervised segmentation of ultrasonic liver images by multiresolution fractal feature vector, Inform. Sci., 175 (2005), 177-199. 6

[14] S. Liu, X.-G. Che, Z.-X. Wang, Improvement of escape time algorithm by no-escape-point, J. Comput., 6 (2011), 16481653. 1

[15] S. Liu, X.-C. Cheng, W. Fu, Y.-P. Zhou, Q.-Z. Li, Numeric characteristics of generalized M-set with its asymptote, Appl. Math. Comput., 243 (2014), 767-774. 6

[16] S. Liu, X.-C. Cheng, C.-H. Lan, W. Fu, J.-T. Zhou, Q.-Z. Li, G.-L. Gao, Fractal property of generalized M-set with rational number exponent, Appl. Math. Comput., 220 (2013), 668-675. 1, 4

[17] S. Liu, W. Fu, H.-M. Deng, C.-H. Lan, J.-T. Zhou, Distributional fractal creating algorithm in parallel environment, Int. J. Distrib. Sens. Netw., 2013 (2013), 8 pages. 1

[18] M. Liu, S. Liu, W. Fu, J.-T. Zhou, Distributional escape time algorithm based on generalized fractal sets in cloud environment, Chin. J. Electron., 24 (2014), 124-127. 1

[19] X.-D. Liu, Z.-L. Zhu, G.-X. Wang, W.-Y. Zhu, Composed accelerated escape time algorithm to construct the general Mandelbrot sets, Fractals, 9 (2001), 149-153. 1 
[20] B. B. Mandelbrot, The fractal geometry of nature, Schriftenreihe für den Referenten, [Series for the Referee] W. H. Freeman and Co., San Francisco, Calif., (1982). 1, 1, 4

[21] P. Melin, O. Castillo, An intelligent hybrid approach for industrial quality control combining neural networks, fuzzy logic and fractal theory, Inform. Sci., 177 (2007), 1543-1557. 6

[22] W. Nazeer, S. M. Kang, M. Tanveer, A. A. Shahid, Fixed point results in the generation of Julia and Mandelbrot sets, J. Inequal. Appl., 2015 (2015), 16 pages. 1

[23] A. Negi, M. Rani, Midgets of superior Mandelbrot set, Chaos Solitons Fractals, 36 (2008), 237-245. 1, 5.1

[24] G. Pastor, M. Romera, G. Alvarez, D. Arroyo, F. Montoya, On periodic and chaotic regions in the Mandelbrot set, Chaos Solitons Fractals, 32 (2007), 15-25. 1, 5.1

[25] K. W. Shirriff, An investigation of fractals generated by $z \rightarrow 1 z^{n}+c$, Comput. Graph., 17 (1993), 603-607. 1

[26] Y.-Y. Sun, K. Hou, X.-D. Zhao, Z.-X. Lu, Research on characteristics of noise-perturbed M-J sets based on equipotential point algorithm, Commun. Nonlinear Sci. Numer. Simul., 30 (2016), 284-298. 1

[27] Y.-Y. Sun, P. Li, Z.-X. Lu, Generalized quaternion M sets and Julia sets perturbed by dynamical noises, Nonlinear Dynam., 82 (2015), 143-156. 1

[28] G. Tiozzo, Topological entropy of quadratic polynomials and dimension of sections of the Mandelbrot set, Adv. Math., 273 (2015), 651-715. 1

[29] D. Wang, S.-T. Liu, Y. Zhao, C.-M. Jiang, Control of the spatial Mandelbrot set generated in coupled map lattice, Nonlinear Dynam., 84 (2016), 1795-1803. 1

[30] W. Xingyuan, Z. Xu, The divisor periodic point of escape-time N of the Mandelbrot set, Appl. Math. Comput., 187 (2007), 1552-1556. 1

[31] J. Yuan, X.-L. Li, Z.-L. Hou, M.-S. Cao, A new escape time algorithm of constructing Julia set, High Technol. Lett., 13 (2007), 194-197. 1 